\title{
Differential Neural Responses during Performance of Matching and Nonmatching to Sample Tasks at Two Delay Intervals
}

\author{
Rebecca Elliott ${ }^{1}$ and Raymond J. Dolan ${ }^{1,2}$ \\ ${ }^{1}$ Wellcome Department of Cognitive Neurology, Institute of Neurology, London WC1N 3BG, United Kingdom, and \\ ${ }^{2}$ Royal Free Hospital School of Medicine, London NW3 2PF, United Kingdom
}

\begin{abstract}
Visual short-term memory in humans and animals is frequently assessed using delayed matching to sample (DMTS) and delayed nonmatching to sample (DNMTS) tasks across variable delay intervals. Although these tasks depend on certain common mechanisms, there are behavioral differences between them, and neuroimaging provides a means of assessing explicitly whether this is underpinned by differences at a neural level. Findings of delay-dependent deficits, after lesions in humans and animals, suggest that the neural implementation of these tasks may also critically depend on the delay interval. In this study we determined whether there were differential neural responses associated with DMTS and DNMTS tasks at two different delay intervals using functional magnetic resonance imaging. Ten healthy volunteers were studied under four test conditions: DMTS and DNMTS at 5 and $15 \mathrm{sec}$ delay. The main effect of DMTS compared with DNMTS across both delay
\end{abstract}

intervals was associated with significant activation in bilateral head of caudate and medial orbitofrontal cortex. By contrast, DNMTS compared with DMTS was associated with significant activation in mediodorsal thalamus, bilateral lateral orbitofrontal cortex, and left premotor cortex. The main effect of short compared with long delay, across both tasks, was associated with significantly greater activity in occipital and parietal cortices. By contrast, long compared with short delay was associated with significantly greater activity in temporal and ventrolateral frontal cortices. We conclude that DMTS and DNMTS are not equivalent and furthermore that the precise neural implementation of these tasks is a dynamic function of delay interval.

Key words: delayed matching to sample; delayed nonmatching to sample; orbitofrontal cortex; caudate; premotor cortex; medial temporal lobe; functional magnetic resonance imaging
Delayed matching to sample (DMTS) and delayed nonmatching to sample (DNMTS) paradigms are widely used to study visual memory in humans and animals. Both tasks require subjects to hold a visual stimulus "on line" over a delay interval before responding to a choice of stimuli. In DMTS, subjects must select the familiar stimulus; in DNMTS they must select the novel stimulus. Successful performance of both tasks depends on the integrity of inferior temporal regions (Gross, 1973; Mishkin, 1982; Horel et al., 1987), posterior perceptual cortex, and medial temporal lobe structures (Zola-Morgan and Squire, 1985; Murray, 1992; Mishkin and Murray, 1994). The entorhinal and perirhinal cortices have been particularly implicated in rats (Mumby and Pinel, 1994; Zhu et al., 1995) and monkeys (Gaffan and Murray, 1992; Meunier et al., 1993). Medial temporal damage in humans also results in various recognition memory deficits (Kimura 1963; Warrington and James 1967; Milner, 1968, 1970). A recent study using a computerized DMTS procedure, analogous to that used in monkeys, reported impairments in patients with temporal lobe lesions or bilateral amygdalohippocampectomies (Owen et al., 1995). There is also evidence implicating prefrontal cortex, particularly ventral and medial regions, in performance of DMTS and DNMTS tasks in monkeys

Received Dec. 8, 1998; revised March 23, 1999; accepted March 23, 1999.

R.J.D. is supported by the Wellcome Trust. We are grateful to Prof. Trevor Robbins (University of Cambridge, Cambridge, UK) for helpful comments and discussion.

Correspondence should be addressed to Dr. Rebecca Elliott, Wellcome Department of Cognitive Neurology, 12 Queen Square, London WC1N 3BG, UK.

Copyright @ 1999 Society for Neuroscience $0270-6474 / 99 / 195066-08 \$ 05.00 / 0$
(Bachevalier and Mishkin, 1986; Meunier et al., 1997) and humans (Milner, 1964; Milner et al., 1985; Owen et al., 1995).

Although common processes are doubtless evoked, and common neural substrates are involved (see above), there are certain key differences between the tasks. For example, Diamond (1991) discussed DNMTS in monkeys and human children as involving memory alone, whereas DMTS involves memory and inhibition of an innate tendency to respond to novel stimuli; thus DMTS is harder than DNMTS in monkeys and infants (Mishkin and Delacour, 1975; Gaffan et al., 1984; Diamond 1991). Interestingly, human adults show the reverse pattern, an important caveat in comparing the monkey and human literature. A primary aim of the present study was to compare explicitly neural responses in DMTS and DNMTS in humans. A further issue is the extent to which different delay intervals influence the neural implementation of DMTS and DNMTS tasks. In monkeys, there is evidence that lesions of temporal lobes (Alvarez et al., 1992, 1995) and prefrontal regions (Fuster, 1995) produce more significant deficits at longer delay intervals. Human patients with Alzheimer's disease or temporal lobe damage show deficits on DMTS tasks with abstract stimuli that become more pronounced at longer delays (Sahakian et al., 1988; Owen et al., 1995; Buffalo et al., 1998). Delay-dependent deficits have also been observed in patients with prefrontal lesions (Owen et al., 1995).

In this study we used functional magnetic resonance imaging (fMRI) to compare evoked activity associated with DMTS and DNMTS at two different delay intervals. We hypothesized that, in addition to activations common to both tasks, there would also be task-specific neural responses. Furthermore, on the basis of recent human and animal data, we predicted that there would be 
Table 1. Percentage of correct behavioral performance under the four memory conditions

\begin{tabular}{lll} 
Condition & $5 \mathrm{sec}[\%(\mathrm{SE})]$ & $15 \mathrm{sec}[\%(\mathrm{SE})]$ \\
\hline DMTS & $92.4(2.2)$ & $91.9(2.8)$ \\
DNMTS & $79.7(4.1)$ & $83.6(3.5)$
\end{tabular}

a difference in the neural substrates at the two delay intervals, with temporal lobe and prefrontal regions more activated at longer delays.

\section{MATERIALS AND METHODS}

MRI scanning technique. Ten healthy right-handed volunteers, 6 male and 4 female, aged 22-40 years, were scanned. A Siemens (Munich, Germany) VISION system at $2 \mathrm{~T}$ was used to acquire both T1-weighted structural images and gradient echo, echo planar T2*-weighted images with blood oxygenation level-dependent (BOLD) contrast. Functional images were acquired in a single run, with a total of 312 volumes acquired for each subject. Each volume comprised $483 \mathrm{~mm}$ axial slices with $3 \mathrm{~mm}$ in-plane resolution, positioned to cover the whole brain. During each run, volumes were acquired continuously every $4.1 \mathrm{sec}$. The run began with six "dummy" volumes to allow for T1 equilibration effects; these volumes were subsequently discarded.

Cognitive activation paradigm. This experiment had a factorial design with four experimental conditions and two control conditions. In all, experimental conditions subjects were presented with a trial-unique test stimulus comprising a complex shape made up of four color subelements (based on stimuli from the Cambridge Automated Neuropsychological Test Battery; Robbins et al., 1994). After $1 \mathrm{sec}$ the test stimulus was replaced by a blank screen. After a delay interval (see below) two choice stimuli were presented, one of which was the original stimulus and the other a novel stimulus. Subjects responded with a button press, pressing either a left or right key depending on whether the left or right stimulus was correct. They were given three seconds in which to make this response (all subjects made all responses within this time) before the next problem appeared. In the "match" conditions, subjects were told to respond by choosing the stimulus that matched the test stimulus, i.e., to select the familiar stimulus. In the "nonmatch" condition, subjects were told to select the novel stimulus. This constituted the first factor. The second factor involved both DMTS and DNMTS tasks being performed under two delay conditions; a "short" interval of $5 \mathrm{sec}$ and a "long" interval of $15 \mathrm{sec}$. Although $15 \mathrm{sec}$ is not truly a long delay in terms of the DMTS and DNMTS literature, and we did not expect significant rates of forgetting, using a substantially longer delay such that significant forgetting occurred would not have been practical in the confines of an fMRI design. If subjects are scanned over a protracted period, discomfort and motion artifacts become serious concerns. There is also an established theoretical framework suggesting that there may be important qualitative differences in human short-term memory at these delay intervals (Phillips, 1974), and the neuropsychological literature on DMTS would support this at a neural level.

Perceptuomotor control conditions. In these conditions subjects saw a random sequence of single stimuli and paired stimuli, none of which matched each other. Subjects were explicitly instructed not to attempt to remember the stimuli and were told to respond by pressing either response button whenever a pair of stimuli appeared. In the short and long control conditions, the temporal spacing of stimuli corresponded to that in the short and long experimental conditions, respectively. This control condition was chosen to match the perceptual and motor demands of the memory task as closely as possible. A consequence of this was to introduce possible confounds, which cannot be addressed in this study. For example, it is not possible to determine empirically the extent to which subjects complied with the instruction not to remember the stimuli. However, by randomizing the order of single and double stimuli, we attempted to obviate any tendency to remember single stimuli. Also, even were this to be a confound, it is not an issue for the key comparisons, which are between different memory conditions and therefore do not involve the control condition at all.

During each activation epoch, three problems, all from the same condition, were presented. Epochs were interleaved with blocks of rest during which subjects were simply required to fixate on a central cross.
The order of conditions was randomized within and between subjects. Given the different delay intervals used, the number of scans per epoch was variable. However, the random effects approach to data analysis described below minimizes the statistical impact of this variability. The alternative to this design was to equate the length of epochs, but this would have meant that subjects completed more short than long trials, which we judged to be a more serious confound.

Data analysis. Data were analyzed using Statistical Parametric Mapping (SPM98; Wellcome Department of Cognitive Neurology) implemented in MATLAB 5 (Mathworks, Sherborn, MA) and run on a SPARC workstation (Sun Microsystems, Surrey, UK). Scans were realigned using the first as a reference and were subsequently transformed into a standard space corresponding to the stereotactic atlas of Talairach and Tournoux (1988) using Montreal Neurological Institute templates. These normalized images were smoothed with a $10 \mathrm{~mm}$ full-width, half-maximum isotropic Gaussian kernel. Analysis was performed using a random effects model with a delayed boxcar waveform. In this model, the relatively higher intersubject, compared with intrasubject, variability is taken into account by first reducing each subject's data to a single mean image for each condition and then using these mean images as the basis for intersubject comparisons. Subject-specific low-frequency drift in signal was removed by modeling with low-frequency sine and cosine waves, and global changes were removed by proportional scaling (Holmes et al., 1998). Effects at each and every voxel were estimated, and regionally specific effects were compared using linear contrasts. The resulting set of voxel values for each contrast constituted a statistical parametric map of the $t$ statistic (SPM $\{t\}$ ), which was then transformed to the unit normal distribution (SPM $\{Z\})$. Statistical inferences were based on the theory of random Gaussian fields (Friston et al., 1995). The stereotactic coordinates of Talairach and Tournoux (1988) are used to report the activation foci, but descriptions of the anatomical localization of the foci were determined using individual structural MRIs of the group and the atlas of Duvernoy (1991).

\section{RESULTS}

\section{Performance data}

The mean percentages of problems solved correctly under the four memory conditions are shown in Table 1 . These data were analyzed using a two-by-two factorial ANOVA. The only significant effect was a main effect of DMTS compared with DNMTS,
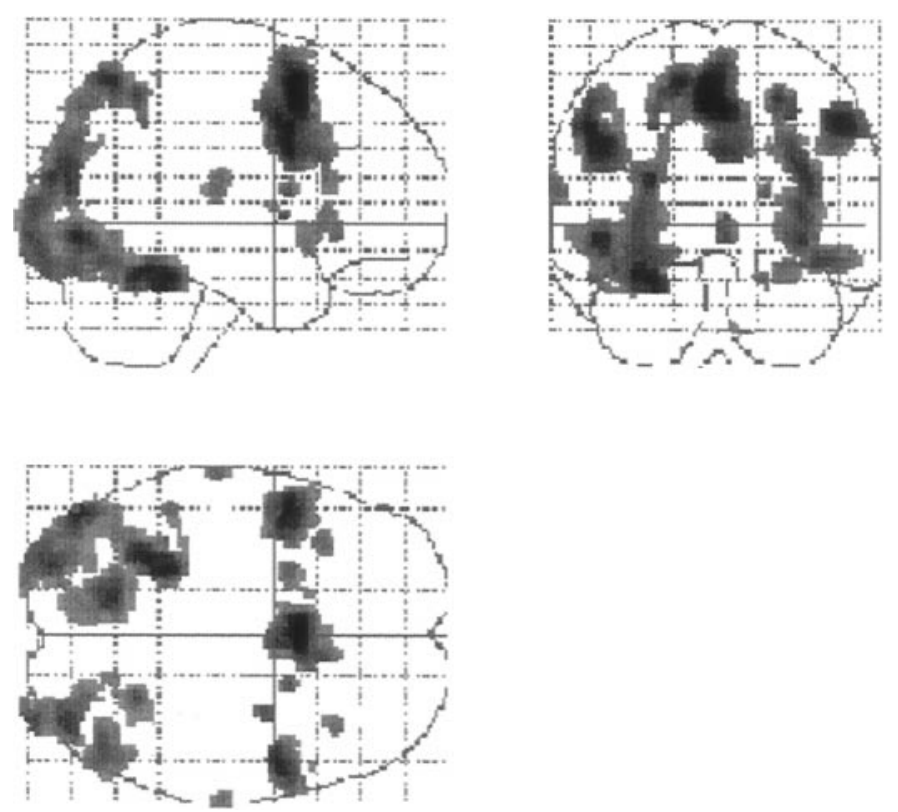

Figure 1. Activations associated with all four memory conditions compared with the perceptuomotor control condition in the form of a statistical parametric map of the $t$ statistic (after transformation to an $\operatorname{SPM}\{Z\}$ ) thresholded at uncorrected $p<0.001$. 


\begin{tabular}{|c|c|c|c|c|c|c|}
\hline \multirow[b]{2}{*}{ Area } & \multirow[b]{2}{*}{ Left/right } & \multirow[b]{2}{*}{ Brodmann's area } & \multicolumn{3}{|c|}{$\begin{array}{l}\text { Talairach coordinates of voxel of } \\
\text { maximal activity }\end{array}$} & \multirow[b]{2}{*}{$Z$ value $^{a}$} \\
\hline & & & $x$ & $y$ & $z$ & \\
\hline \multicolumn{7}{|l|}{ Memory-control } \\
\hline \multirow[t]{2}{*}{ Anterior cingulate } & $\mathrm{L}$ & 32 & -4 & 10 & 54 & 4.97 \\
\hline & $\mathrm{R}$ & 24 & 6 & 12 & 30 & 4.63 \\
\hline \multirow[t]{2}{*}{ Middle frontal gyrus } & $\mathrm{L}$ & 9 & -52 & 8 & 40 & 4.47 \\
\hline & $\mathrm{R}$ & 9 & 52 & 2 & 42 & 4.37 \\
\hline \multirow[t]{2}{*}{ Medial occipital gyrus } & $\mathrm{R}$ & 19 & 38 & -82 & 18 & 3.90 \\
\hline & $\mathrm{L}$ & 19 & -34 & -96 & 8 & 3.68 \\
\hline Superior parietal cortex & $\mathrm{R}$ & 7 & 26 & -54 & 52 & 3.78 \\
\hline Posteror fusiform gyrus & $\mathrm{R}$ & $18 / 19$ & 46 & -68 & -12 & 3.56 \\
\hline Cerebellum & $\mathrm{L}$ & & -26 & -42 & -48 & 4.37 \\
\hline \multicolumn{7}{|l|}{ Control-memory } \\
\hline Cuneus & $\mathrm{R} / \mathrm{L}$ & 19 & -4 & -90 & 24 & 4.33 \\
\hline Medial parietal cortex & $\mathrm{R}$ & 7 & 12 & -54 & 34 & 4.13 \\
\hline Middle temporal gyrus & $\mathrm{R}$ & 21 & 64 & -46 & -4 & 4.02 \\
\hline Anterior frontal cortex & $\mathrm{R}$ & 10 & 20 & 60 & 22 & 3.76 \\
\hline Angular gyrus & $\mathrm{L}$ & 39 & -46 & -68 & 32 & 3.84 \\
\hline \multirow[t]{2}{*}{ Cerebellar vermis } & $\mathrm{L}$ & & -30 & -70 & -46 & 3.68 \\
\hline & $\mathrm{R}$ & & 30 & -80 & -36 & 3.82 \\
\hline
\end{tabular}

${ }^{a} Z$ value threshold is 3.09 (corresponding to $p<0.001$ uncorrected).

with subjects performing better in the DMTS condition $\left(F_{(1,36)}=\right.$ 4.6; $p<0.05)$.

\section{Relative activations associated with all memory conditions compared with control}

When all four memory conditions (DMTS and DNMTS across both delays) were compared with the perceptuomotor control conditions, significant evoked neural activity changes were seen in bilateral anterior cingulate (BA 32 and 24; significant at $p<0.05$ corrected) and bilateral posterior middle frontal gyrus (BA 9), bilateral medial occipital gyrus (BA 19), right superior parietal cortex (BA 7), right posterior fusiform gyrus (BA 18), and left cerebellar hemisphere (all at $p<0.001$ uncorrected; Fig. 1, Table 2).

Reductions in evoked activity relative to control (all $p<0.001$, uncorrected) were seen in cuneus (BA 19), right medial parietal cortex (BA 7), right lateral medial temporal gyrus (B 21), bilateral anterior frontal cortex (BA 10), left angular gyrus (BA 39), and the cerebellar vermis bilaterally (Table 2).

When the memory conditions were compared with the baseline fixation condition, a very similar pattern of evoked activity was seen as for the comparison with perceptuomotor control. However the reverse comparison, fixation compared with memory, showed no significant changes, which suggests that the reductions in evoked activity for memory relative to the perceptuomotor control were attributable to evoked activity in the control condition rather than decreased activity in the memory conditions per se.

\section{Relative activations associated with DMTS compared with DNMTS}

Significant evoked neural activity associated with DMTS compared with DNMTS was observed in the head of caudate nucleus on the left extending to the subgenual cingulate cortex (BA 24) and medial orbitofrontal cortex (BA 25 extending to 11) $(p<$ 0.05 corrected; Fig. 2, Table 3). Neural responses at a lower level of significance ( $p<0.001$ uncorrected) were seen in corresponding regions on the right.

\section{Relative activations associated with DNMTS compared with DMTS}

Significant evoked neural activity ( $p<0.001$ uncorrected) associated with DNMTS compared with DMTS was seen in left premotor cortex (BA 6), lateral orbitofrontal cortex bilaterally (BA 11) (Fig. 3), left dorsomedial thalamus, and left precuneus (BA 7) (Fig. 3, Table 3).

\section{Correlations between neural response and behavioral performance}

Because subjects performed significantly worse at DNMTS than DMTS, this raises the question of whether any of the activations specific to the individual tasks simply reflect this performance difference. To address this we repeated the above contrasts, modeling performance as a covariate of interest. There were no significant correlations between neural response and performance in any of the regions identified in the between-task comparisons.

\section{Relative activations associated with short compared with long delay intervals}

Short, compared with long, delays were associated with significant evoked activity in medial and inferior occipital gyri (BA 19), fusiform gyrus (BA 37), and cerebellar hemispheres, all bilaterally $(p<0.05$ corrected). There was also evoked activity in superior parietal cortex (BA 7) bilaterally ( $p<0.001$ uncorrected; Fig. 4, Table 4).

\section{Relative activations associated with long compared with short delay intervals}

Long compared with short delays were associated with significant evoked activity in temporal pole bilaterally (BA 21/38), medial temporal gyrus (BA 21) bilaterally, and inferior temporal gyrus 

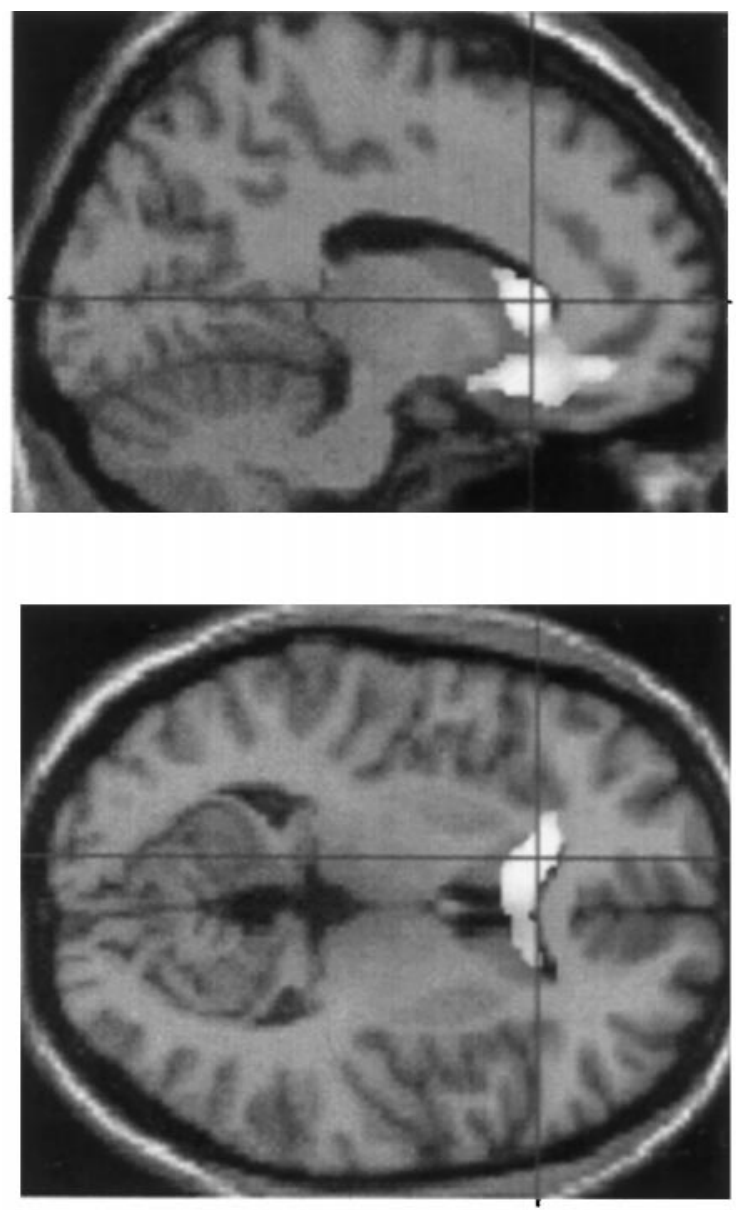

Figure 2. Relative activations associated with DMTS compared with DNMTS. The statistical parametric map of the $t$ statistic (after transformation to an $\operatorname{SPM}\{Z\}$ ) thresholded at $p<0.001$ is shown rendered onto a standard MRI template and focused on the left head of caudate $(x=$ $-12 ; y=24 ; z=8)$.

(BA 20) and left hippocampus (all $p<0.05$ corrected). There was also activation of inferior frontal gyrus (BA 44/45) bilaterally at $p<0.001$ uncorrected (Fig. 4, Table 4).

\section{Activations relative to control seen for both DMTS and DNMTS}

Common task-related activations were determined from a conjunction analysis of DMTS compared with control and DNMTS compared with control (using separate blocks of the control task for each comparison) and defines areas commonly activated in both tasks. Significant evoked activity (at $p<0.001$ uncorrected) was seen in anterior cingulate (BA 32) bilaterally, posterior medial frontal gyrus (BA 9) bilaterally, medial occipital gyrus (BA 19) bilaterally, left posterior parietal cortex (BA 7), and left cerebellum.

\section{Modulation of activations associated with DMTS compared with DNMTS by different delays}

These comparisons represent interaction terms in the factorial design. Activations of bilateral caudate and medial orbitofrontal cortex, associated with DMTS compared with DNMTS, were significantly greater at short than long delays $(p<0.001$ uncorrected). Adjusted neural responses in caudate are shown in Figure 5. Activation of precuneus, associated with DNMTS compared with DMTS, was significantly greater at short than long delays ( $p<0.05$ corrected) and there was also a significant activation of right amygdala ( $p<0.05$ corrected) not seen in the main effect expressed solely in DNMTS at short delays. The right anterior cingulate (BA 32), although activated in all four conditions, showed augmented activation for DNMTS at long delays $(p<0.001$ uncorrected).

\section{DISCUSSION}

The key findings of this study are of significant differences in neural response to DMTS and DNMTS tasks and, furthermore, significant differences in neural response to short and long delays. DMTS was associated with greater activation in caudate and medial orbitofrontal cortex. By contrast, DNMTS was associated with activation in premotor cortex, lateral orbitofrontal cortex, and mediodorsal thalamus. Comparisons between 5 and $15 \mathrm{sec}$ delay intervals showed that neural responses in posterior perceptual regions were greater at shorter delays, whereas responses in temporal lobes and ventrolateral frontal cortices were greater at longer delays. Activations common to all four conditions were seen in anterior cingulate, medial frontal gyrus, and various
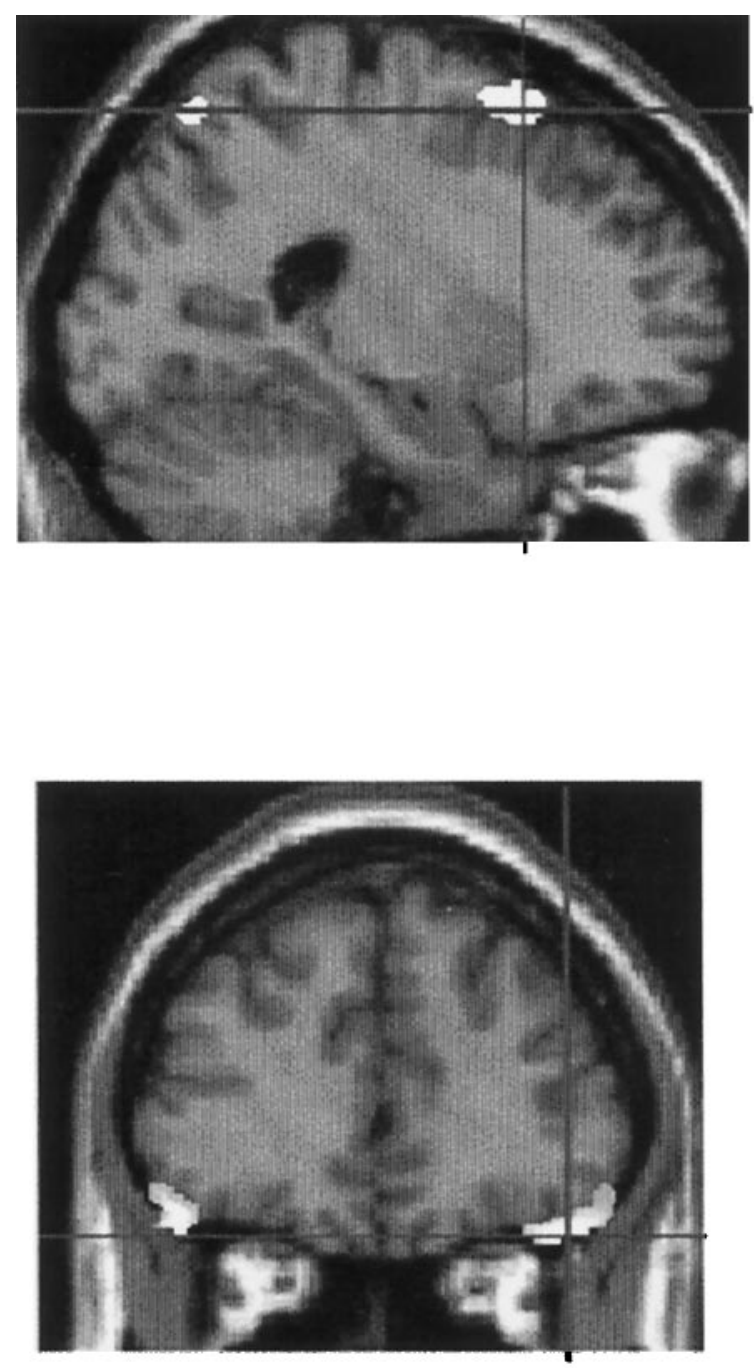

Figure 3. Relative activations associated with DNMTS compared with DMTS. The statistical parametric map of the $t$ statistic (after transformation to an $\operatorname{SPM}\{Z\}$ ) thresholded at $p<0.001$ is shown rendered onto a standard MRI template, focused on the premotor $(x=-22 ; y=18 ; z=$ $60)$ and lateral orbitofrontal $(x=46 ; y=44 ; z=-22)$ activations. 
Table 3. Areas where evoked activity was significantly different for DMTS and DNMTS

\begin{tabular}{|c|c|c|c|c|c|c|}
\hline \multirow[b]{2}{*}{ Area } & \multirow[b]{2}{*}{ Left/right } & \multirow[b]{2}{*}{ Brodmann's area } & \multicolumn{3}{|c|}{$\begin{array}{l}\text { Talairach coordinates of voxel of } \\
\text { maximal activity }\end{array}$} & \multirow[b]{2}{*}{$Z$ value } \\
\hline & & & $x$ & $y$ & $z$ & \\
\hline \multicolumn{7}{|l|}{ DMTS-DNMTS } \\
\hline \multirow[t]{2}{*}{ Caudate nucleus } & $\mathrm{L}$ & & -12 & 24 & 8 & 5.36 \\
\hline & $\mathrm{R}$ & & 12 & 14 & 16 & 4.25 \\
\hline Subgenual cingulate & $\mathrm{L}$ & 24 & -6 & 26 & 12 & 4.86 \\
\hline \multirow[t]{2}{*}{ Medial orbitofrontal cortex } & $\mathrm{L}$ & 25 & -10 & 18 & -16 & 4.79 \\
\hline & $\mathrm{R}$ & 25 & 12 & 20 & -16 & 4.70 \\
\hline \multicolumn{7}{|l|}{ DNMTS-DMTS } \\
\hline Premotor cortex (SMA) & $\mathrm{L}$ & 6 & -22 & 18 & 60 & 4.31 \\
\hline \multirow[t]{2}{*}{ Lateral orbitofrontal cortex } & $\mathrm{R}$ & 11 & 46 & 44 & -22 & 4.28 \\
\hline & $\mathrm{L}$ & 11 & -48 & 36 & -22 & 3.45 \\
\hline Dorsomedial thalamus & $\mathrm{L}$ & & -4 & -10 & 10 & 3.92 \\
\hline Medial parietal cortex (precuneus) & $\mathrm{L}$ & 7 & -20 & -66 & 60 & 4.09 \\
\hline
\end{tabular}

Table 4. Areas where significant evoked activity differed for the two delay intervals

\begin{tabular}{|c|c|c|c|c|c|c|}
\hline \multirow[b]{2}{*}{ Region of activation } & \multirow[b]{2}{*}{ Left/right } & \multirow[b]{2}{*}{ Brodmann's area } & \multicolumn{3}{|c|}{ Talairach coordinates } & \multirow[b]{2}{*}{$Z$ value } \\
\hline & & & $x$ & $y$ & $z$ & \\
\hline \multicolumn{7}{|l|}{$5-15 \mathrm{sec}$} \\
\hline \multirow{2}{*}{ Medial occipital gyrus } & $\mathrm{L}$ & 19 & -26 & -90 & 16 & 5.47 \\
\hline & $\mathrm{R}$ & 19 & 20 & -86 & 16 & 4.22 \\
\hline \multirow[t]{2}{*}{ Inferior occipital gyrus } & $\mathrm{L}$ & 19 & -42 & -80 & 14 & 5.57 \\
\hline & $\mathrm{R}$ & 19 & 42 & -92 & 0 & 6.07 \\
\hline \multirow[t]{2}{*}{ Fusiform gyrus } & $\mathrm{R}$ & 37 & 32 & -58 & -18 & 5.32 \\
\hline & $\mathrm{L}$ & 37 & -40 & -64 & -18 & 5.02 \\
\hline \multirow[t]{2}{*}{ Cerebellum } & $\mathrm{R}$ & & 36 & -76 & -18 & 6.05 \\
\hline & $\mathrm{L}$ & & -26 & -86 & -26 & 4.76 \\
\hline Medial parietal cortex & $\mathrm{L} / \mathrm{R}$ & 7 & -8 & -62 & 58 & 4.00 \\
\hline \multicolumn{7}{|l|}{$15-5 \mathrm{sec}$} \\
\hline \multirow[t]{2}{*}{ Temporal pole } & $\mathrm{L}$ & 38 & -50 & 4 & -32 & 5.81 \\
\hline & $\mathrm{R}$ & 21 & 44 & 12 & -34 & 4.09 \\
\hline \multirow[t]{2}{*}{ Middle temporal gyrus } & $\mathrm{L}$ & 21 & -50 & -8 & -14 & 5.01 \\
\hline & $\mathrm{R}$ & 21 & 58 & -22 & -12 & 4.38 \\
\hline Inferior temporal gyrus & $\mathrm{L}$ & 20 & -34 & 0 & -36 & 4.33 \\
\hline Hippocampus & $\mathrm{L}$ & & -28 & -16 & -18 & 4.04 \\
\hline \multirow[t]{2}{*}{ Inferior frontal gyrus } & $\mathrm{R}$ & $44 / 45$ & 58 & 22 & 12 & 4.06 \\
\hline & $\mathrm{L}$ & $44 / 45$ & -56 & 18 & 14 & 3.80 \\
\hline
\end{tabular}

posterior cortical regions. These regions overlap with those reported in earlier positron emission tomography (Swartz et al., 1994; Faillenot et al., 1997; Elliott and Dolan 1998) and fMRI (Courtney et al., 1996, 1997; Klingberg et al., 1997) studies using DMTS or related tasks.

The finding of differential neural responses to DMTS and DNMTS explicitly confirms that these tasks are not equivalent at the neural implementational level in humans. At debriefing, subjects reported their approach to DNMTS as identical to DMTS up until the final stage of response execution. For both tasks they attempted to remember the sample during the delay and identified the familiar stimulus at the choice stage. DNMTS diverges from DMTS in that rather than responding to this familiar stimulus, they respond to the other. On the basis of these subjective reports, it is plausible that DNMTS involves an additional processing stage to DMTS, necessitating inhibition of responses to familiar stimuli. It should be noted that this is the reverse of the situation in monkeys (Gaffan et al., 1984) and human infants (Diamond, 1991) in which the instinctive preference is for novel objects, so that DMTS rather than DNMTS involves the extra inhibition stage.

The premotor activation in DNMTS is consistent with this theory, because premotor cortex is associated with response inhibition in monkeys (Sawaguchi, 1996) and humans (Casey et al., 1997; Kiefer et al., 1998). However, ventral regions of the prefrontal cortex have most reliably been associated with response inhibition (Jones and Mishkin, 1972; Kowalska et al., 1991; Fuster, 1997), in accord with our observation of lateral orbitofrontal activation. Monkeys with lateral orbitofrontal lesions fail to inhibit responding on no-go trials of a go/no-go task (Iversen and Mishkin, 1970). In an fMRI study using a go/no-go task, Casey et al. (1997) demonstrated activation in several prefrontal regions, 
A
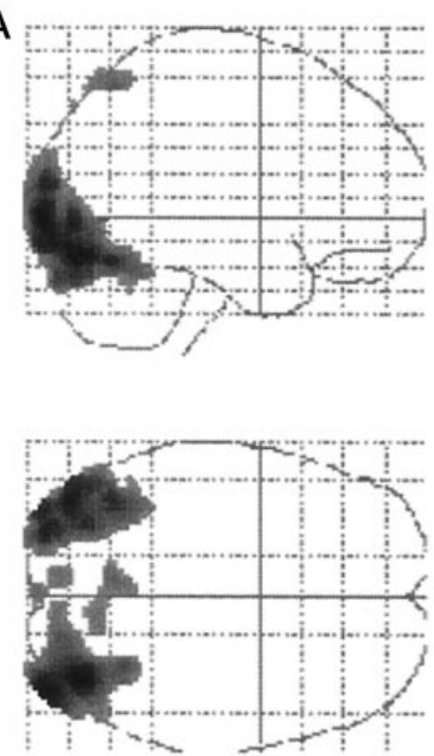

B
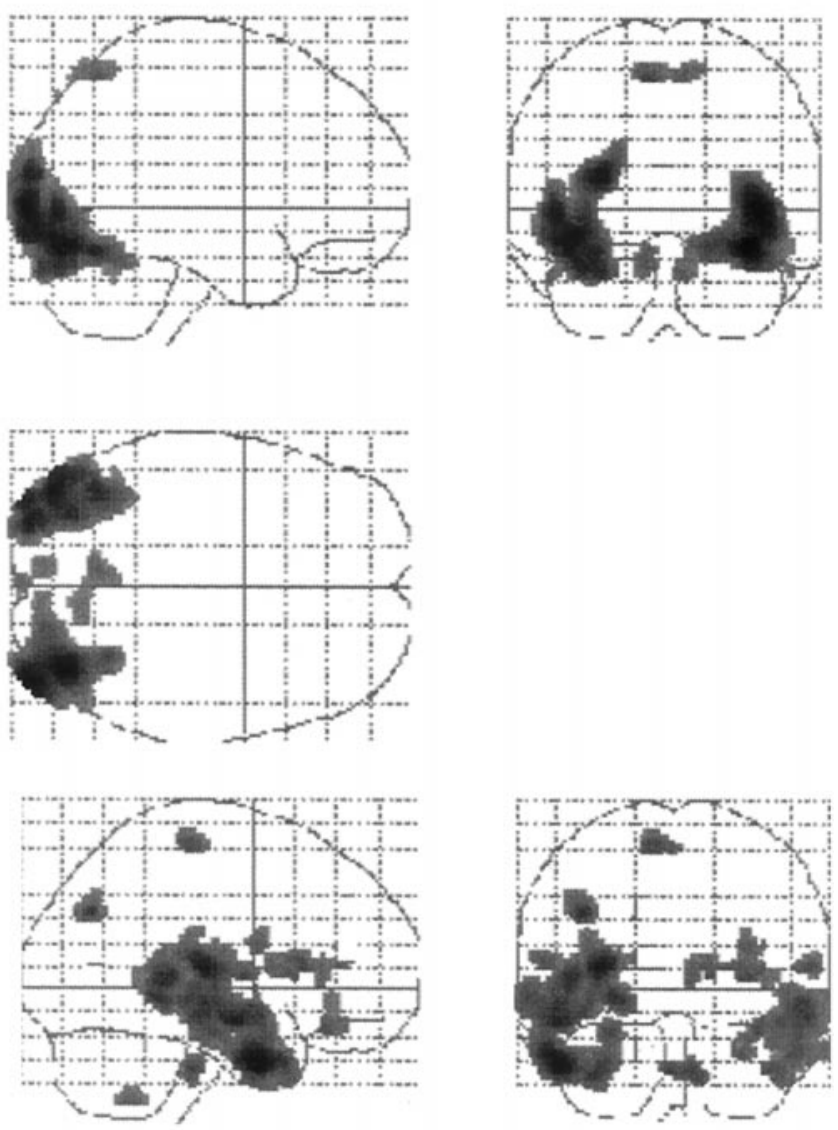

related (Rushworth et al., 1997), although it should be noted that this study lesioned inferior convexity rather than orbitofrontal cortex. Interestingly Rushworth et al. (1997) used DMTS rather than DNMTS; however, as discussed, in monkeys it is DMTS that involves the additional inhibition stage.

DMTS compared with DNMTS was associated with activation of caudate and medial orbitofrontal cortex. The caudate has previously been implicated in animals performing various delayed response tasks with similarities to the paradigm used here (Divac, 1967; Levy et al., 1997). Human patients with basal ganglia disorders show deficits on delayed response tasks (Partiot et al., 1996) and a computerized DMTS task similar to that used in the present study (Sahakian et al., 1988; Owen et al., 1993; A. D. Lawrence, personal communication). These deficits are independent of delay interval, consistent with our finding that caudate activation does not increase at the longer delay. It has been suggested (Packard and White, 1991; MacDonald and White, 1993) that the dorsal striatum is particularly involved in memory tasks with a win-stay component, in which a key requirement is to make stimulus-response associations. Similarly, Rolls (1994) has argued that caudate neurons respond to stimuli that signal behavioral responses. Medial orbitofrontal cortex has also been implicated in tasks requiring subjects to associate stimuli with appropriate behavioral responses (Bechara et al., 1994; Rolls et al., 1994). It has been argued, based on primate lesion studies, that the involvement of medial orbitofrontal cortex in DMTS and DNMTS may be attributable to learning of associations between stimuli and rewarded responses (Meunier et al., 1997).

Thus, a possible explanation for caudate and medial orbitofrontal activations associated with DMTS, but not DNMTS, is that they represent neural responses to stimuli that specify the actual response. Although these tasks are more complex than classic stimulus-response habit tasks, they do involve a component of mapping stimuli to correct responses. In DMTS, as soon as subjects see the sample stimulus, they know the stimulus they must subsequently respond to. By contrast, in DNMTS the sample stimulus specifies a nonresponse. Thus in DMTS there is a greater association between a stimulus, or its representation during the delay, and the specification of the correct behavioral response. Neural responses in the caudate and medial orbitofrontal cortex may mediate this association. These activations are modulated by delay, being greater at shorter delays, maybe re-

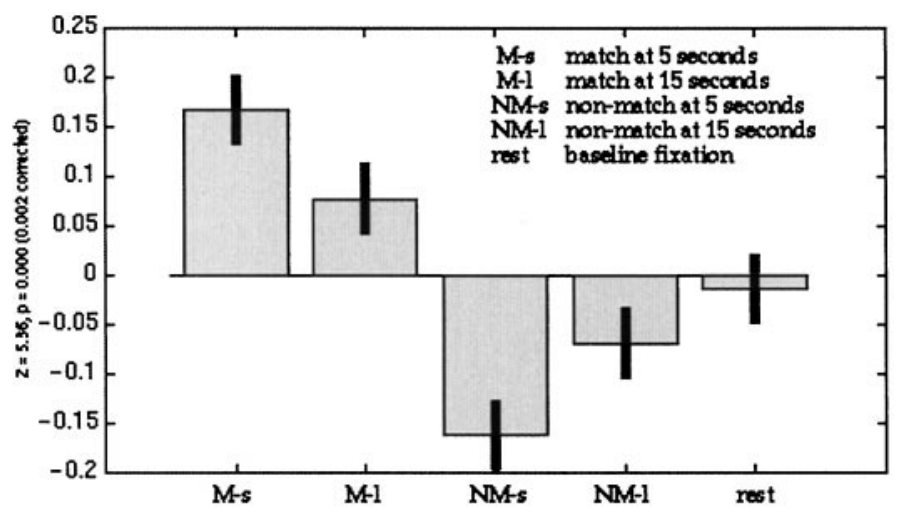

Figure 5. Adjusted neural response in the head of caudate under the four different conditions and low-level rest. The adjusted response is based on normalized counts, and this graph shows the relative counts associated with each condition. Because the numbers represent relative counts, there

are no associated units. consistent with the observation that ventral prefrontal lesions in primates impair components of matching tasks that are not delay- the form of statistical parametric maps of the $t$ statistic (after transformation to an $\operatorname{SPM}\{Z\}$ ) thresholded at $p<0.001$. $A$, Regions more activated at short delays; $B$, regions more activated at long delays.

including BA 11, a region also activated in our present study. Casey et al. (1997) also found that activation of this region, but not dorsolateral prefrontal cortex, was significantly correlated with behavioral performance. Thus, more successful inhibition of inappropriate responses predicted greater activity in this region. There is also evidence that damage to the mediodorsal thalamus in humans produces deficits that may be interpreted in terms of response inhibition (Leng and Parkin, 1988; Joyce and Robbins, 1991; Forstl and Sahakian, 1993). The contention that the prefrontal and thalamic activations are associated with response inhibition in our study is supported, to some extent, by an absence of delay-related modulation of activity in these regions. If activations were delay-related, such modulation would be expected. The lack of delay dependency in lateral orbitofrontal cortex may be 
flecting the fact that stimulus-response mapping is strongest with closer temporal linkage. Also, as we will argue, at short delays representation of a more veridical form of the stimulus, rather than a relatively abstract description, is linked to the required response.

Our other main finding was the distinct pattern of activation associated with varying delay intervals. Although both 5 and 15 sec would be considered within the scope of "short-term" memory, there are pronounced differences in the associated neural substrates. At $5 \mathrm{sec}$, greater activation is seen in occipital and medial parietal cortices relative to $15 \mathrm{sec}$ delays. At $15 \mathrm{sec}$, greater activation is seen in temporal lobes and ventrolateral frontal cortices. The temporal lobe activations include medial temporal structures of hippocampus and neighboring rhinal cortices that have been strongly implicated in primate studies of DMTS (Gaffan and Murray, 1992; Meunier et al., 1993). Our delay-dependent findings are consistent with observations in animals that both temporal and frontal lobe lesions produce more severe deficits on DMTS and DNMTS as delay increases (Alvarez et al., 1992, 1995; Bauer and Fuster, 1976). They also accord with observations that in humans with temporal or frontal lesions, there is a trend toward more pronounced DMTS impairment at longer delays (Owen et al., 1995). Patients with dementia of the Alzheimer type, characterized by pronounced temporal lobe pathology, also show delay-dependent DMTS deficits (Sahakian et al., 1988).

The differential neural responses at the two delay intervals suggest different processing requirements under these conditions. The predominant perceptual cortex activations at shorter delays suggest a greater dependence on primarily visual representations of the stimulus during the delay interval. Phillips (1974) characterized a distinct short-term visual memory system in which recent visual events are actively visualized and persist for delays of up to $10 \mathrm{sec}$, in the absence of intervening distracters. Memory over longer delays requires conversion of visual representations into more structural descriptions. Our findings suggest that such a short-term posticonic memory system may be subsumed by posterior cortical perceptual systems. Once the delay exceeds the limits of this system, other regions are recruited to mediate more abstract representations. Consistent with this account is subjects' reported reliance on a "snapshot" approach at short delays, whereas at longer delays, they rehearsed a verbal description of the stimuli. Increased activation in temporal inferior frontal cortices would accord with a greater reliance on verbal coding. However, it should be noted that increasing delay also implicates these regions more strongly in monkey studies, and therefore it seems unlikely that verbal mediation is the only factor involved.

In conclusion, this study provides interesting insights into the neural substrates of classic short-term memory tasks. We have demonstrated that, although there are common substrates that mediate DMTS and DNMTS in humans, there are also pronounced differences. We have proposed that the differential activations seen in DNMTS compared with DMTS may be associated with the requirement to inhibit prepotent responses to familiar stimuli. Activations in the reverse comparison may reflect associations between representations of the test stimulus and anticipated behavioral response. The neural substrates of both tasks depend critically on the delay period, with performance at shorter delays dependent on early perceptual cortices, whereas longer delays involve temporal and frontal cortices, perhaps reflecting more abstract stimulus representation.

\section{REFERENCES}

Alvarez P, Zola-Morgan S, Squire LR (1992) Impairment of long-term memory and sparing of short-term memory in monkeys with medial temporal lobe lesions: a response to Ringo [comment]. Behav Brain Res $52: 1-5$.

Alvarez P, Zola-Morgan S, Squire LR (1995) Damage limited to the hippocampal region produces long-lasting memory impairment in monkeys. J Neurosci 15:3796-3807.

Bachevalier J, Mishkin M (1986) Visual recognition impairment following ventromedial but not dorsolateral prefrontal lesions in monkeys. Behav Brain Res 20:249-261.

Bauer RH, Fuster JM (1976) Delayed matching and delayed response deficit from cooling prefrontal cortex in monkeys. J Comp Physiol Psychol 90:293-302.

Bechara A, Damasio AR, Damasio H, Anderson SW (1994) Insensitivity to future consequences following damage to human prefrontal cortex. Cognition 50:7-15.

Buffalo EA, Reber PJ, Squire LR (1998) The human perirhinal cortex and recognition memory. Hippocampus 330-339.

Casey BJ, Trainor RJ, Orendi JL, Schubert AB, Nystrom LE, Giedd JN, Castellanos X, Haxby JV, Noll DC, Cohen JD, Forman SD, Dahl RE, Rapoport JL (1997) A developmental functional MRI study of prefrontal activation during performance of a g-no-go task. J Cognit Neurosci 9:835-847.

Courtney SM, Ungerleider LG, Keil K, Haxby JV (1996) Object and spatial visual working memory activate separate neural systems in human cortex. Cereb Cortex 6:39-49.

Courtney SM, Ungerleider LG, Keil K, Haxby JV (1997) Transient and sustained activity in a distributed neural system for human working memory. Nature 386:608-611.

Diamond A (1991) Guidelines for the study of brain-behaviour relationships during development. In: Frontal lobe function and dysfunction (Levin HS, Eisenberg HM, Benton AL, eds), pp 339-380. Oxford: Oxford UP.

Divac I, Rosvold HE, Sewarebart MK (1967) Behavioural effects of selective ablation of the caudate nucleus. J Comp Physiol Psychol 63:184-190.

Duvernoy HM (1991) The human brain: surface, three-dimensional sectional anatomy and MRI. New York: Springer.

Elliott R, Dolan RJ (1998) The neural response in short-term visual recognition memory for perceptual conjunctions. NeuroImage 71:14-22.

Faillenot I, Sakata H, Costes N, Decety J, Jeannerod M (1997) Visual working memory for shape and 3D-orientation: a PET study. NeuroReport 8:859-862.

Forstl H, Sahakian BJ (1993) Thalamic radiodensity and cognitive performance in mild and moderate dementia of the Alzheimer type. J Psychiatry Neurosci 18:33-37.

Friston KJ, Holmes AP, Worsley KJ, Poline J-B, Frith CD, Frackowiak RSJ (1995) Statistical parametric maps in functional imaging: a general approach. Hum Brain Mapp 2:189-210.

Fuster JM (1995) Memory in the cerebral cortex: an empirical approach to neural networks in the human and nonhuman primate. Cambridge, MA: MIT.

Fuster JM (1997) The prefrontal cortex. Anatomy, physiology and neuropsychology of the frontal lobe, Ed 3. New York: Raven.

Gaffan D, Muray EA (1992) Monkeys (Macaca fascicularis) with rhinal cortex ablations succeed in object discrimination learning despite 24-hr retention intervals and fail at matching to sample despite double sample presentations. Behav Neurosci 106:30-38.

Gaffan D, Gaffan EA, Harrison S (1984) Effects of fornix transection on spotaneous and trained non-matching by monkeys. Q J Exp Psychol $36 \mathrm{~b}: 285-303$.

Gross CG (1973) Visual functions of the inferotemporal cortex. In: Handbook of sensory physiology (Jung R, ed), pp 451-482. Berlin: Springer.

Holmes AP, Josephs O, Buechel C, Friston KJ (1998) Statistical modelling of low frequency confounds in fMRI. NeuroImage 5:S480.

Horel JA, Pytko-Joiner DE, Voytko ML, Salisbury K (1987) The performance of visual tasks while segments of the inferotemporal cortex are suppressed by cold. Behav Brain Res 23:29-42.

Iversen S, Mishkin M (1970) Perseverative interference in monkey fol- 
lowing selective lesions of the inferior prefrontal convexity. Exp Brain Res 11:376-386.

Jones B, Mishkin M (1972) Limbic lesions and the problem of stimulusreinforcement associations. Exp Neurol 36:362-377.

Joyce EM, Robbins TW (1991) Frontal lobe function in Korsakoff and non-Korsakoff alcoholics: planning and spatial working memory. Neuropsychologia 29:709-723.

Kiefer M, Marzinzik F, Weisbrod M, Schherg M, Spitzer M (1998) the time course of brain activations during response inhibition: evidence from event-related potentials in a go/no-go task. NeuroReport 9:765-770.

Kimura D (1963) Right temporal lobe damage: perception of unfamiliar stimuli after damage. Arch Neurol 8:264-271.

Klingberg T, O'Sullivan BT, Roland PE (1997) Bilateral activation of fronto-parietal networks by incrementing demand in a working memory task. Cereb Cortex 7:465-471.

Kowalska DM, Bachevalier J, Mishkin M (1991) The role of the inferior prefrontal convexity in performance of delayed nonmatching to sample. Neuropsychologia 29:583-600.

Leng NRC, Parkin AJ (1988) Double dissociation of frontal dysfunction in organic amnesia. Br J Clin Psychol 27:359-362.

Levy R, Friedman HR, Davachi L, Goldman-Rakic PS (1997) Differential activation of the caudate nucleus in primates performing spatial and non-spatial working memory tasks. J Neurosci 17:3870-3882.

McDonald RJ, White NM (1993) A triple dissociation of memory systems: hippocampus, amygdala and dorsal striatum. Behav Neurosci 107:3-22.

Meunier M, Bachevalier J, Mishkin M, Murray EA (1993) Effects on visual recognition of combined and separate ablations of the entorhinal and perirhinal cortex in rhesus monkeys. J Neurosci 13:5418-5432.

Meunier M, Bachevalier J, Mishkin M (1997) Effects of orbital frontal and anterior cingulate lesions on object and spatial memory in rhesus monkeys. Neuropsychologia 35:999-1016.

Milner B (1964) Some effects of frontal lobectomy in man. In: The frontal granular cortex and behaviour (Warren JA, Akert K, eds), pp 313-334. New York: McGraw-Hill.

Milner B (1968) Visual recognition and recall after right temporal lobe excision in man. Neuropsychologia 6:191-209.

Milner B (1970) Memory and medial temporal regions of the brain. In: Biology of memory (Pribram KH, Broadbent DE, eds). New York: Academic.

Milner B, Petrides M, Smith ML (1985) Frontal lobes and the temporal organisation of memory. Hum Neurobiol 4:137-142.

Mishkin M (1982) A memory system in the monkey. Philos Trans R Soc Lond B Biol Sci 298:85-92.

Mishkin M, Delacour J (1975) An analysis of short-term visual memory in the monkey. J Exp Psychol Anim Behav Process 1:326-334.

Mishkin M, Murray EA (1994) Stimulus recognition. Curr Opin Neurobiol 4:200-206.

Mumby DG, Pinel JPJ (1994) Rhinal cortex lesions and object recognition in rats. Behav Neurusci 108:1-8.

Murray EA (1992) Medial temporal lobe structure contribute to recognition memory: the amygdaloid complex versus the rhinal cortex. In:
The amygdala: neurobiological aspects of emotion, memory and mental dysf unction (Aggleton JP, ed), pp 453-470. New York: Wiley.

Owen AM, Bekinska M, James M, Leigh PN, Summers BA, Quinn NP, Sahakian BJ, Robbins TW (1993) Visuospatial memory deficits at different stages of Parkinson's disease. Neuropsychologia 31:627-644.

Owen AM, Sahakian BJ, Semple J, Polkey CE, Robbins TW (1995) Visuo-spatial short term recognition memory and learning after temporal lobe excisions, frontal lobe excisions or amygdalohippocampectomy in man. Neuropsychologia 33:1-24.

Packard MG, White NM (1991) Dissociation of hippocampus and caudate nucleus memory systems by post-training intracerebral injection of dopamine agonists. Behav Neurosci 105:295-306.

Partiot A, Verin M, Pillon B, Teixeira-Ferreira C, Agid Y, Dubois B (1996) Delayed response tasks in basal ganglia lesions in man: further evidence for a striato-frontal cooperation in behavioural adaptation. Neuropsychologia 34:709-721.

Phillips WA (1974) On the distinction between sensory storage and short term visual memory. Percept Psychophys 16:283-290.

Robbins TW, James M, Owen AM, Sahakian BJ, McInnes L, Rabbitt P (1994) Cambridge Neuropsychological Battery (CANTAB): a factor analytic study of a large sample of normal elderly volunteers. Dementia 5:266-281.

Rolls ET (1994) Neurophysiology and cognitive functions of the striatum. Rev Neurol 150:648-660.

Rolls ET, Hornak J, Wade D, McGrath J (1994) Emotion related learning in patients with social and emotional changes associated with frontal lobe damage. J Neurol Neurosurg Psychiatry 57:1518-1524.

Rushworth MFS, Nixon PD, Eacott MJ, Passingham RE (1997) Ventral prefrontal cortex is not essential for working memory. J Neurosci 17:4829-4838.

Sahakian BJ, Morris RG, Evenden JL, Heald A, Levy R, Philpot M, Robbins TW (1988) A comparative study of visuospatial memory and learning in Alzheimer-type dementia and Parkinson's disease. Brain 111:695-718.

Sawaguchi T (1996) Functional modular organization of the primate prefrontal cortex for representing working memory process. Cognit Brain Res 5:157-163.

Swartz BE, Halgren E, Simpkins F, Fuster JM, Mandelkern M, Kristadumkorn T, Gee M, Brown C, Ropchan JR, Blahd WH (1994) An 18FDG-PET study of cortical activation during a short-term visual memory task in humans. NeuroReport 5:925-928.

Talairach J, Tournoux P (1988) Coplanar stereotactic atlas of the human brain. Stuttgart: Thieme.

Warrington E, James M (1967) An experimental investigation of facial recognition in patients with unilateral cerebral lesions. Cortex 3:317-326.

Zhu XO, Brown MW, Aggleton JP (1995) Neuronal signalling of information important to visual recognition memory in rat rhinal and surrounding cortices. Eur J Neurosci 7:753-765.

Zola-Morgan S, Squire LR (1985) Medial temporal lesions in monkeys impair memory on a variety of tasks sensitive to human amnesia. Behav Neurosci 99:22-34. 